Ann. Génét. Sél. anim., I980, 12 (I), I-7.

\title{
Hérédité des variations de couleur du pelage du Buffle africain (Syncerus caffer)
}

\author{
J. J. LAUVERGNE et C. RENVOISÉ * \\ Département de Génétique animale \\ Centre national de Recherches zootechniques, I.N.R.A., \\ 78350 Jouy-en-Josas \\ * Muséum national d'Histoire naturelle, Parc Zoologique, Paris XII
}

\section{Résumé}

La sous-espèce des savanes du Buffle africain (Syncerus Caffer caffer) est noire, la sous-espèce de forêts (Syncerus caffer nanus) rouge à extrémités noires. L'examen de croisements faits au Muséum national d'Histoire naturelle de Paris montre que le phénotype croisé est intermédiaire, c'est-à-dire rouge à extrémités noires avec une extension plus marquée des plages noires que chez nanus. On peut penser que les deux sous-espèces diffèrent par un allèle au locus Agouti A : caffer étant probablement récessif $a a$ cependant que nanus porterait l'allèle $A \times$ pour le rouge à extrémités noires, leur formule au locus $\mathrm{E}$ d'Extension étant la même : $E^{+} E^{+}$.

Dû sans doute à sa distribution géographique étendue en Afrique (cf fig. I), le Buffe sauvage africain présente une grande variété morphologique qui a incité les anciens auteurs à décrire de nombreuses espèces ou races (MAMMERICKS, r960). Toutefois, plus récemment, les taxonomistes se sont accordés à considérer le Bufle africain comme monophylétique : (Syncerus caffer) avec deux types extrêmes : Syncerus caffer caffer (le Buffle du Cap) et Syncerus caffer nanus le Buffe nain de forêt (DORST et DANDELOT, I970).

En fait cette conception est peut-être tout de même trop simplificatrice car les examens caryologiques révèlent une différence dans les garnitures chromosomiques : $2 n=54$ pour nanus; $2 n=52$ pour caffer (ATLAS OF CHROMOSOMES, I969, r970).

Ces deux variétés - disons peut-être mieux, sous-espèces — diffèrent en outre par la taille, le cornage et la couleur.

Syncerus caffer, caffer qui vit dans les savanes, est de grande taille (jusqu'à $\mathrm{I}, 70 \mathrm{~m}$ au garrot) noir de pelage avec des cornes qui lui recouvrent le chignon (fig. 2). 


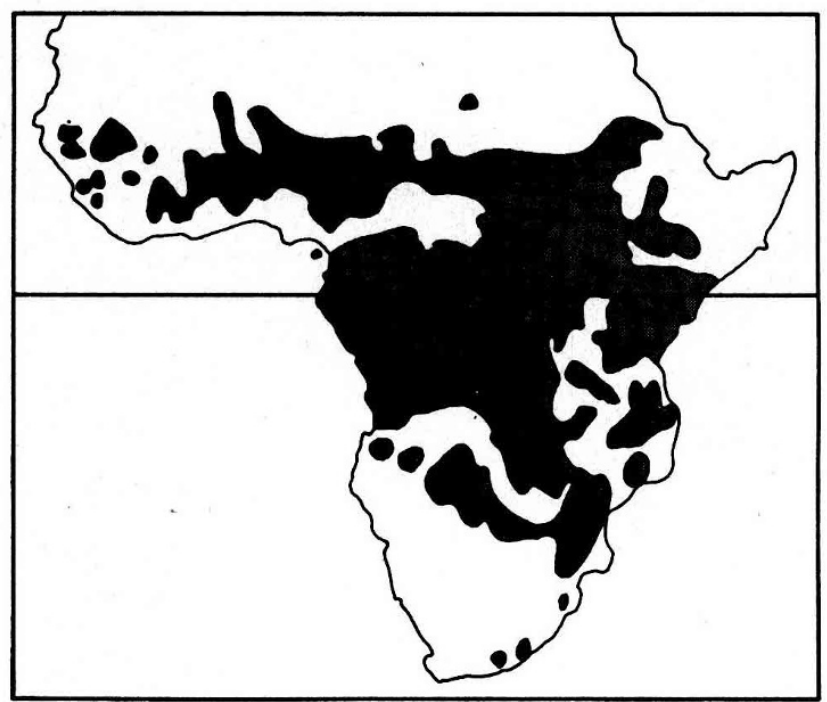

FIG. I. - Répartition géographique du Buffle afvicain (Syncerus caffer).

(d'après DORST et DANDELOT, 1970).

Geographic repartition of the African Buffalo (Syncerus caffer).

(from DORST and DANDELOT, I970).

Syncerus caffer nanus, qui vit dans la forêt équatoriale et dont l'habitat est en voie de réduction, est plus petit et son pelage est fauve à extrémités noires. Les cornes sont plus petites et écartées (fig. 2).

Depuis longtemps le Muséum national d'Histoire naturelle de Paris possède des sujets de ces sous-espèces et des croisements ont eu lieu au Parc Zoologique de Paris ce qui nous permet d'aborder dans le présent article le déterminisme héréditaire de la couleur dans cette espèce qui a été très peu étudiée sous ce rapport (ANSELL, I975) alors que la couleur du Buffle asiatique (Bubalus bubalis) qui présente de nombreuses souches domestiquées est mieux connue (cf SEARLE, I968 et Mason, I974 pour revue).

\section{Matériel et méthode}

\section{A. - Précisions sur la coloration du pelage de Syncerus caffer}

On a vu que la couleur du pelage de la variété de savane (Syncerus caffer caffer) est uniformément noire. Toutefois ce pelage est assez clairsemé et laisse voir une peau qui, par endroits, à l'instar de celle de certaines variétés asiatiques de Bubalus bubalis (Ross-CoCKRILI, I974) est fortement mélanisée, présentant une couleur gris ardoisé (fig. $2 a$ et $b$ ).

Le pelage du corps de la variété de forêt ( $S$. caffer nanus) est fauve tirant sur le roux, (un peu comme la race bovine Limousine) avec, aux extrémités, des plages noires : sur le chanfrein, sur le cou, dans les oreilles, sur les pattes (qui sont bottées de noir connue chez la vache Tarine). Le toupillon est également noir (fig. $2 b$ et $c$ et fig. 3 ). 

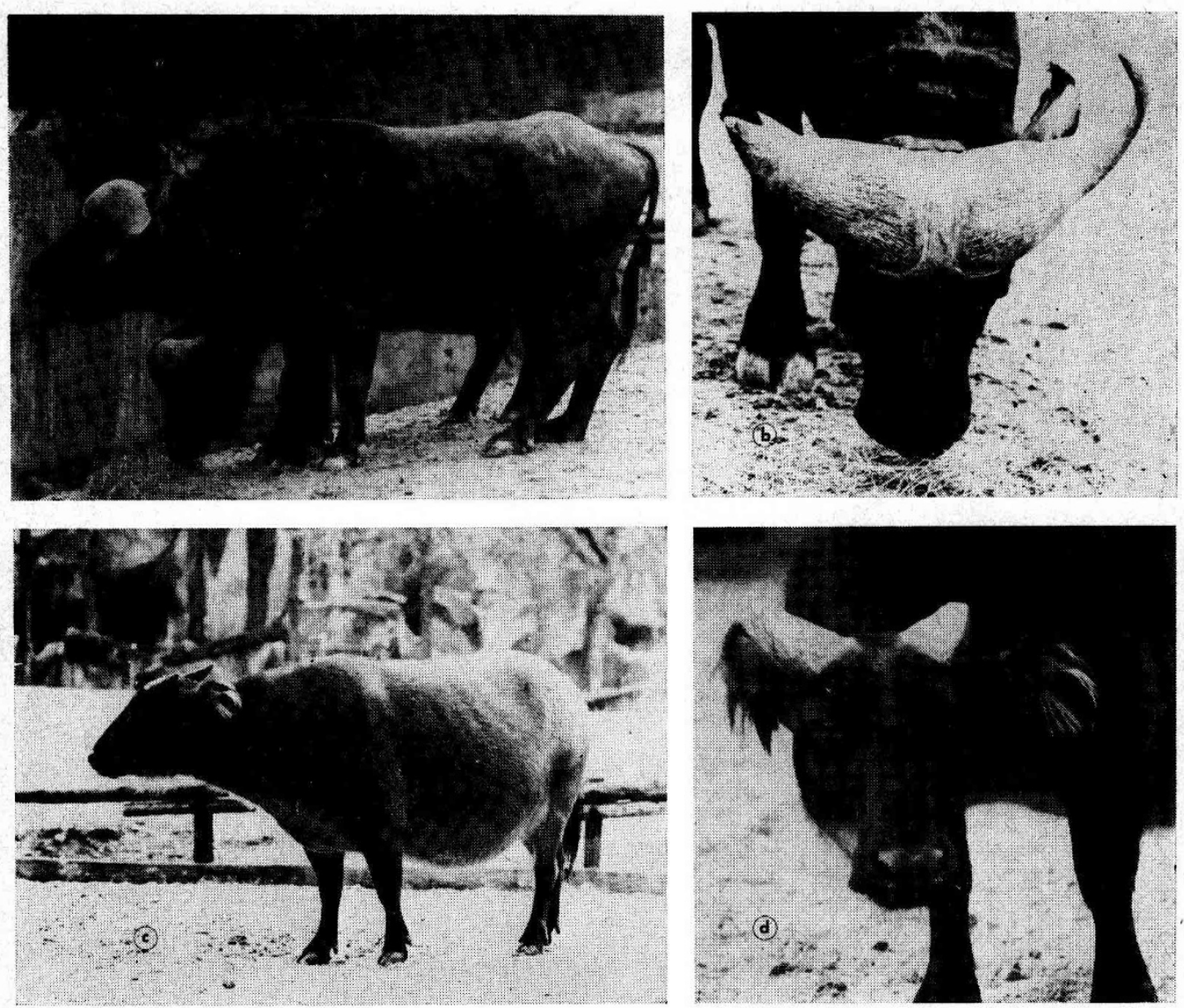

FIG. 2.

a) Syncerus caffer caffer (var. equinoctialis) mâle $n^{\circ} 4669$. Le poil est noir mais l'animal est presque glabre spécialement sur la croupe, la peau est gris ardoisé.

b) Le même animal vu de face. On note le cornage en casque cachant le chignon caractéristique de cette sous-espèce.

c) Syncerus caffer nanus femelle no $n^{\circ} 7^{7}$ originaire du Gabon. On distingue bien les extrémités noires et on note la densité du pelage plus grande que chez caffer.

d) Même animal qu'en c, vu de face avec le cornage caractéristique chez nanus: cornes petites et écartées laissant voir le chignon.

a) Syncerus caffer caffer (var. equinoctialis) mâle $\mathrm{n}^{\circ}{ }_{4669}$. The hair coat is black but hair are rare and even lacking in some parts. The skin is slate grey.

b) Same animal as in a) one notes the horns in helm shape hiding the poll.

c) Syncerus caffer nanus female $n^{\circ}$ I $47_{70}$ originating from Gabon. One can see the black extremities. The hair coat is more dense as in caffer.

d) Same animal as in c) facing with the typical horns of this variety : small and apart with a visible poll.

L'extension du noir est variable d'un individu à l'autre et, semble-t-il, augmente avec l'âge. La peau, que l'on aperçoit à travers un pelage moins clairsemé que dans la variété de savane mais tout de même moins dense que chez les bovins des régions tempérées, est mélanisée, même dans les zones recouvertes de poils, sauf dans la partie déclive du corps. 


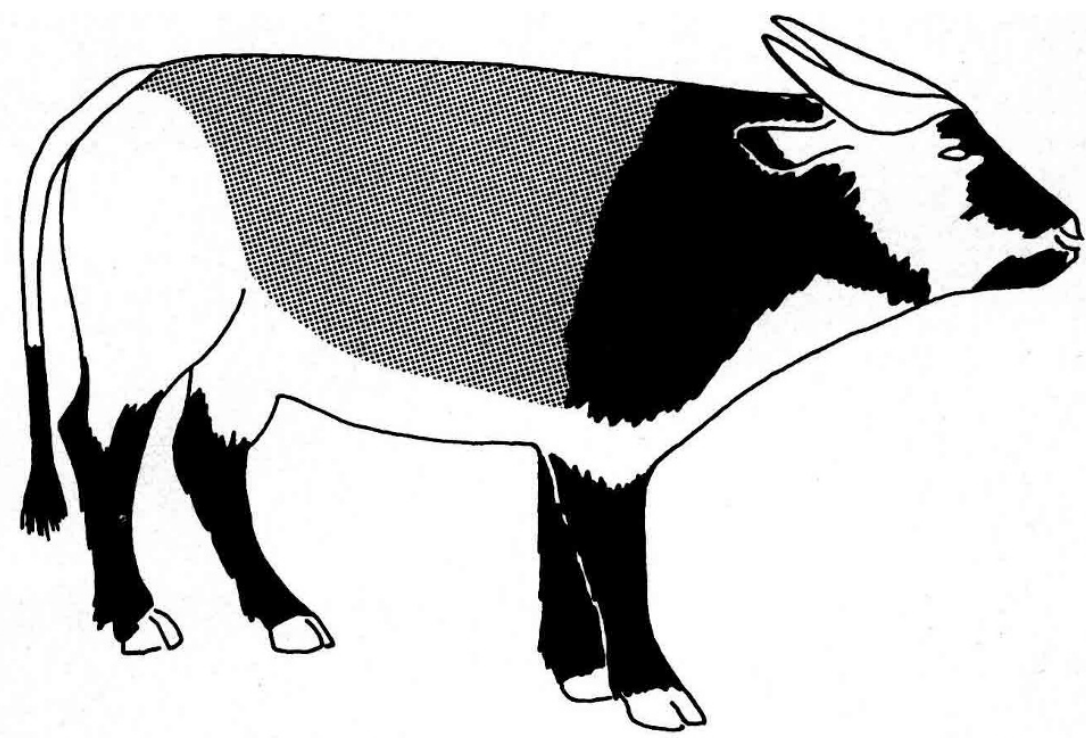

FIG. 3. - Extensions minimum et maximum des marques noires sur le pelage de Syncerus caffer nanus, le Buffle Nain des forêts d'Afrique.

Minimum and maximum extension of black markings on the coat of Syncerus caffer nanus, the dwarf Forest Buffalo of Africa.

\section{B. - Les croisements pratiqués}

La première naissance de croisés namus $\times$ caffer remonte à 1954 . Depuis, il y a eu 5 autres produits de croisements, le dernier étant né en 1979.

\section{Résultats}

Six produits ont été obtenus : 2 FI, 2 back cross (un sur chaque parent) et 2 croisés back cross $\times$ nanus.

Ces croisements sont présentés dans la figure 4 .

Les FI étaient presque glabres, à la manière du parent caffer, mais la coloration de leur pelage rappelait celle du parent nanus rouge à extrémités noires, avec une extension moins poussée du rouge (fig. 2). Dans les croisements suivants on retrouvait les formes parentales bien rouge ou bien noir.

\section{Discussion}

Nos données méritent sans doute d'être présentées vu la rareté des connaissances sur le Buffe africain. Elles ne permettent pas cependant d'interpréter définitivement en terme génétique, les variations colorées observées, tout au 


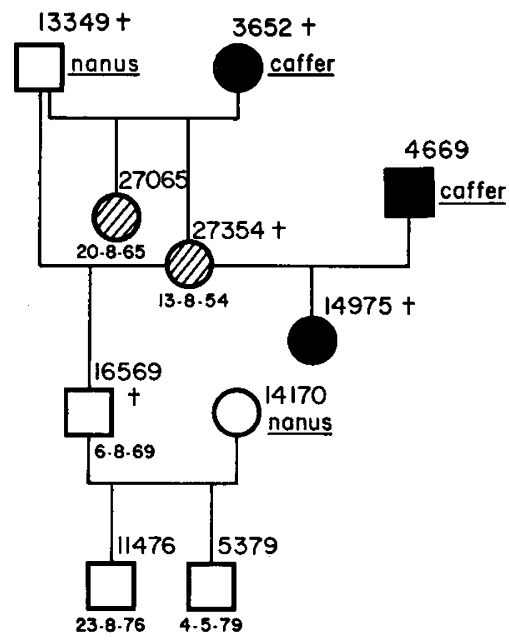

FIG. 4. - Relations de parenté entre les croisés nanus $\times$ caffer obtenus au Parc Zoologique de Paris. La date de naissance est indiquée pour les animaux nés au Zoo. Les animaux marqués d'une croix avaient disparu en date du 30 avril 1980.

Pedigrees of the crosses nanus $\times$ caffer born in the Parc Zoologique of Paris. The bivthdate of those born in the Zoo is given underneath. All the animals already away april 301980 are quoted with a cross.

o Femelle de phénotype nanus (rouge à extrémités noires).

Female of nanus phenotype (red with black extremities).

- Femelle de phénotype caffer (noir).

Female of caffer phenotype (black).

ธ Mâle de phenotype nanus (rouge à extrémités noires).

Male of nanus phenotype (red with black extremites).

- Male de phénotype caffer (noir).

Male of caffer phenotype (black).

En hachuré les phénotypes intermédiaires.

In hachury the intermediary phenotypes.

plus, peut-on avancer une hypothèse qui s'appuie sur des considérations d'homologie avec les espèces de mammifères mieux connus génétiquement.

Selon cette hypothèse, la sous-espèce nanus porterait un allèle autosomal pour le rouge à extrémités noires au locus $A$ gouti (A). Cet allèle serait dominant plus ou moins complètement sur les autres allèles de la série et en particulier sur 1'allèle le plus récessif de tous, celui du noir récessif.

Cette hypothèse nous semble préférable à 1'hypothèse alternative qui consisterait à supposer que le noir est déterminé par un allèle dominant de la série Extension (E) qui, chez les Mammifères, est épistatique sur les génotypes de la série Agouti et qui semble rendre compte du noir observé chez Bos taurus (LAUVERGNE, I966; SEARLE, I968). En effet, le noir dominant et épistatique a, en général, une épistasie totale alors que nous observons chez le Buffle une dominance intermédiaire qui rappelle certaines interactions observées au sein de la série Agouti chez d'autres Ruminants (mouton, Adalsternsson, I970; chèvre, I,AUVERGNE, I976).

En outre, cette dominance incomplète de l'allèle pour le rouge à extrémités 
noires - que l'on pourrait noter $A^{y}$ (à l'instar de Bos taurus, LAUVERGNE, I966) sur le noir que l'on pourrait noter $a$, peut s'expliquer par l'action de gènes modificateurs qui se sont accumulés chez la variété caffer de savane pour augmenter la mélanisation d'un corps devenu presque glabre. Bien que les deux systèmes de mélanocytes pour colorer la peau et pour colorer le poil soient, dans une certaine mesure, indépendants, dans ces conditions extrêmes il n'est pas impossible qu'un tel phénomène - spécialement intéressant à étudier car les espèces de mammifères glabres ou quasi glabres sont rares - ait pu jouer.

Le rouge à extrémités noires existe chez Bos taurus comme nous venons de le voir, mais également chez le chien où l'interprétation génétique n'est toutefois pas définitive (LITTLE, I957; SEARLE, I968) et chez le cheval où il serait induit par 1'allèle bai en $A$ gouti (symbole $A$ ou $A^{+}$selon les auteurs; Geeurts, I977).

L'existence de ces variations colorées au sein d'une espèce sauvage qui, comme le Buffle d'Afrique, occupe plusieurs niches écologiques, s'explique assez bien.

On a pu avoir au départ un animal fauve à extrémités noires et à pelage dense qui même vivant en savane d'altitude là où le rayonnement solaire est intense, aurait pu, de ce fait, garder sa livrée comme beaucoup d'espèces d'antilopes. Mais peut-être pour des raisons d'adaptation à la chaleur et aux mœurs aquatiques, le Buffe africain - à l'instar d'ailleurs d'autres espèces vivant entre les tropiques, (éléphant, rhinocéros) - a perdu une partie de son pelage laissant la peau sans protection contre les U.V. Cette dernière a dî alors se pigmenter pour résister à ce rayonnement comme nous l'avons vu plus haut, même chez la forme nanus où le pelage est encore rouge. Supposons alors qu'advienne une mutation de noir récessif au locus $A$ gouti, courante dans toutes les espèces de mammifères. Cela a pu être pour la forme de savane très exposée au rayonnement solaire une occasion de renforcer son aptitude à la mélanisation, d'où sa généralisation chez caffer, cependant que la variété de forêt nanus (en régression d'ailleurs avec la régression de la forêt) continue à porter la livrée d'origine de l'espèce et que, dans les zones de contact entre les deux sous-espèces on trouve un mélange des patrons colorés (ANSELL, I975).

\section{Conclusion}

La variété de savane du Buffe sauvage africain, Syncerus caffer caffer est presque noire de poil et de peau avec, vraisemblablement, la formule aa au locus A gouti cependant que la variété de forêt (Syncerus caffer nanus) porterait la formule $A^{y} A^{y}$ ( $A^{y}$ fauve à extrémités noires) qui pourrait être la formule ancestrale de l'espèce.

Reçu pour publication en mai I98o.

\section{Remerciements}

Le manuscrit de cet article a été lu par les Professeurs. DrEUx, Zoologie Ecole Normale Supérieure et RINJARD, sous-directeur du Parc Zoologique de Paris. Leurs commentaires et critiques nous ont beaucoup aidés. 


\section{Summary}

\section{Heredity of coat colour variation in the African Buffalo (Syncerus caffer)}

The "savana "subspecies of the African Buffalo (Syncerus Caffer caffer) is black the "forest " subspecies (Syncerus caffer manus) is red with black extremities. The $\mathrm{F}_{1}$ which were obtained at the Museum d'Histoive Ncturelle of Paris were intermediary, red but with a greater extension of the black areas. One can think than the two subspecies differ from one another in one allele at the locus Agouti $A$ : caffer could probably be $a a$, nünus carrying the $A^{\mathrm{y}}$ allele for the red with black extremities. The formula of both at the $E$ locus could be the same : $E^{+} E^{+}$.

\section{Références bibliographiques}

Adalsternsson S., 1970. Colour inheritance in Icelandic sheep and relation between colour, fertility and fertilization. $J$. Agr. Res. Icel., 2, 3-135.

ANSELI, W.F.H., I975. Colour patterns in Africa. The $A r k, 2$ (8), $201-202$.

ATLAS OF CARYOTYPES, I969. 3, I45; I970, 4, I92.

DoRst J., DANDELOT P., I970. The larger mammals of Africa. Collins, London.

GeUR's R., I977. Hair colour in the Horse. J. A. Allen, London, ro8 p.

LAUVERGNE J. J., I966. Génétique de la couleur du pelage des bovins domestiques. Bibliog $r$. Genet., 20 I, 68.

Lauvergne J. J., I978. Gènes de coloration du pelage des chèvres Alpines chamoisées et Poitevines. Ann Génét. Sél. amni., 10, I81-190.

LITTLE C. C., I957. The inheritance of coat color in dogs. Comstock, Ihaca, New York.

Mammericks M., 1960. Le Buffle (Monographie du genre Bubalus). Bull. Agric. Congo Belge Ruanda-Urundi, 51, I 7 I-2 I 2 .

Mason I. L., I974. Genetics, in Ross CockrILI, W., The Husbandry and Health of the Domestic Buffalo, FAO, Rome, 57-87.

ROSS-COCKRILL, W., I974. Observations on skin colour and hair patterns, in RosS COCKRILL, W. The Husbandry and Health of the Domestic Buffalo, FAO, Rome, 48-56.

SEARLE A. G., I968. Comparative Genetics of Coat colour in Mammals. Logos Press, Academic Press, New-York, London. 